\title{
Antiresonance in switched systems with only unstable modes
}

\author{
Maurizio Porfiri॰* \\ Center for Urban Science and Progress, Department of Mechanical and Aerospace Engineering, \\ and Department of Biomedical Engineering, New York University, Tandon School of Engineering, Brooklyn, New York 11201, USA \\ Russell Jeter $\odot$ \\ Department of Mathematics and Statistics, Georgia State University, P.O. Box 4110, Atlanta, Georgia 30302-410, USA \\ Igor Belykh $\oplus^{\dagger}$ \\ Department of Mathematics and Statistics, Georgia State University, P.O. Box 4110, Atlanta, Georgia 30302-410, USA \\ and Department of Control Theory, Lobachevsky State University of Nizhny Novgorod, 23 Gagarin Avenue, 603950 Nizhny Novgorod, Russia
}

(Received 9 October 2020; revised 2 December 2020; accepted 23 February 2021; published 1 April 2021)

\begin{abstract}
Antiresonance is a key property of dynamical systems that leads to the suppression of oscillations at select frequencies. We present the surprising example of a switched system that alternates between unstable modes, but exhibits antiresonance for a wide range of switching frequencies. We elucidate the stabilization mechanism and characterize the range of antiresonant frequencies for periodic and stochastic switching. The demonstration of antiresonance in a minimalistic variation of the Stuart-Landau model opens the door for a new paradigm in the study and design of switched systems.
\end{abstract}

DOI: 10.1103/PhysRevResearch.3.L022001

Introduction. Switched dynamics are pervasive in theoretical physics, neuroscience, and engineering [1]. For example, the temporal patterning of interactions in active matter discontinuously evolves as their comprising units change their spatial organization [2-5]. Likewise, synchronization in brain networks emerges from sporadic, on-off synaptic interactions between spiking and bursting neurons [6]. Switch logic is also ubiquitous in engineering systems, such as converters and communication networks $[7,8]$.

Switched systems consist of multiple modes, each with autonomous dynamics $[9,10]$. Stability of the modes does not univocally determine the stability of a switched system: A number of analytical studies documented a critical role of the switching frequency [11-15], which was also registered in applications to complex systems [16-22]. Notably, switched systems with only stable modes can become unstable at select switching frequencies $[23,24]$. This phenomenon can be viewed as an internal resonance [25], wherein the switching frequency does not allow the dynamics in each of the stable modes to decay before the onset of a new switch.

Heraclitus' theory of the unity of opposites has seldom defeated our intuition of physical processes, from the particle-wave duality in quantum mechanics [26] to the

\footnotetext{
*Corresponding author: mporfiri@nyu.edu

${ }^{\dagger}$ Corresponding author: ibelykh@gsu.edu

Published by the American Physical Society under the terms of the Creative Commons Attribution 4.0 International license. Further distribution of this work must maintain attribution to the author(s) and the published article's title, journal citation, and DOI.
}

matter/antimatter asymmetry problem [27]. Antiresonance continues to be extensively investigated in acoustics [28], optics [29], biology [30], and quantum physics [31], with a focus on smooth, stable systems. On the other hand, antiresonance of switched systems with only unstable modes has never been documented.

Is it possible to induce antiresonance in the form of stable response of a system switching between two unstable modes? Here, we provide a positive answer to this question by offering the first example of a switched system composed of only unstable modes that displays a stable response in a finite range of switching frequencies. Our system has an unstable average that hinders stability in the fast-switching limit, and the instability of both modes hampers stability for slow-switching frequencies.

Governing equations. We consider a two-dimensional nonlinear switched system with complex state vector $z(t)=$ $x(t)+i y(t)$, alternating between two modes, according to a binary signal $s(t)$ that switches between 0 and 1 . Initially, we focus on a periodic signal of period $T$ with a duty cycle $\delta$, such that the switch is on for $\delta T$ units of time and is off for $(1-\delta) T$ units of time; later, we explore stochastic switching. Our example is a minimalistic variation of a Stuart-Landau oscillator of the form

$$
\begin{aligned}
& s=0: \dot{z}=(1+i \omega) z+i \omega \frac{z^{3}}{|z|^{2}}, \\
& s=1: \dot{z}=(1+i \omega) z-i \omega \frac{z^{3}}{|z|^{2}},
\end{aligned}
$$

where $\omega$ is the radian frequency [32]. 

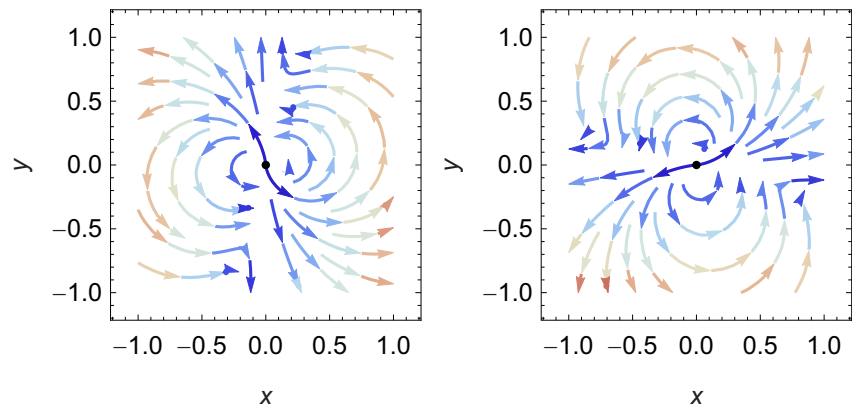

FIG. 1. Streamlines of the two unstable modes of the switched system given in (1) for $\omega=2$, with color indicating the intensity of the vector field for $s=0$ (left) and $s=1$ (right).

The function $f\left(z, z^{*}\right)=z \pm i \omega \frac{z^{3}}{|z|^{2}}$, where $z^{*}$ indicates complex conjugate, introduces rotational noninvariance. Specifically, $f\left(z e^{i \chi}, z^{*} e^{-i \chi}\right) \neq e^{i \chi} f\left(z, z^{*}\right)$ [33]. This symmetry breaking is a key difference from a standard oscillator [34]. Lack of rotational invariance has been extensively investigated in Stuart-Landau models, describing anisotropic Heisenberg spin systems [35] and chimera deaths in networks [36].

Another key difference is the lack of differentiability at the origin, which acts as a hybrid fixed point. Although unstable, this fixed point has features of an unstable node and a stable focus (see Fig. 1). A typical trajectory will first rotate about the origin, reducing its distance from it like a stable focus, and then it will escape to infinity along the principal unstable direction like an unstable node. The principal unstable directions are the $y$ and $x$ axis for the first $(s=0)$ and second $(s=1)$ modes, respectively. The interplay between the contrasting features of the modes is the driver for the antiresonance of the switched system. The nonlinearity in (1) can be viewed as a feedback to the standard oscillator, similar to classical nonlinear feedback systems to manipulate temporal states of single physical oscillators [37] and achieve demand-controlled network synchronization [38].

In principle, one can construct a range of oscillators that possess the same structure as (1) by manipulating the StuartLandau model [33] to capture richer dynamics at the onset of oscillations. Our choice is motivated by the possibility to pursue a completely analytical treatment of the problem.

Slow- and fast-switching limits. In the limit case $T \gg 1$ (the rate of growth of each mode) the switched system is unstable, as it would spend a large fraction of time in one of the unstable modes before it could reswitch to the other [12]. After spiraling toward the origin for almost an entire quadrant, each trajectory will approach the principal unstable direction of the mode and travel away from the origin. Switching will cause the trajectory to experience a sudden turn and after each period, the distance from the origin will increase (see Fig. 2).

An unstable response is also registered for the fastswitching case, in which $T \ll 1$ (see Fig. 2). This result is explained by examining the average system [13], obtained by averaging (1),

$$
\dot{z}=(1+i \omega) z+i(1-2 \delta) \omega \frac{z^{3}}{|z|^{2}} .
$$
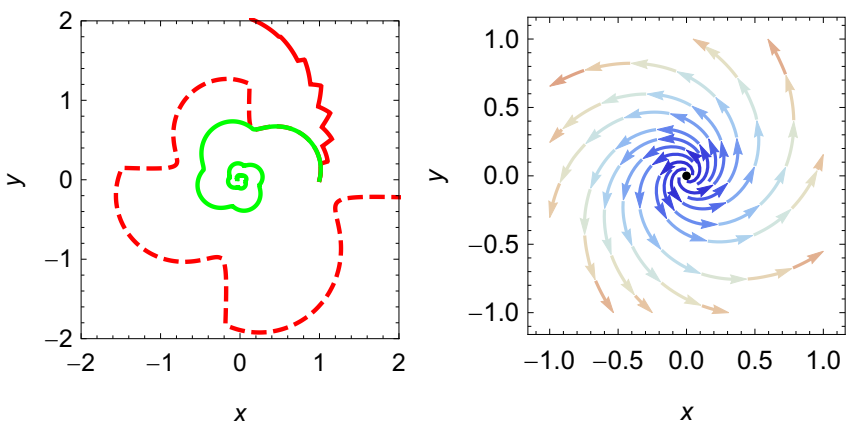

FIG. 2. Trajectories of the periodically-switched system in (1) for $\omega=2$ and $\delta=0.5$ and different values of $T$ (left): $T=0.1$ (solid red), $T=2$ (green), and $T=5$ (dashed red). Initial condition is $(1,0)$ for all cases. Streamlines of the average system in (1) for $\omega=2$ and $\delta=0.5$, with color indicating the intensity of the vector field (right).

Similar to the individual modes, all the trajectories, except for the origin, spiral out to infinity.

Although often used in the analytical treatment of switched systems [12,13], the slow- and fast-switching limits offer a close representation of the switched system when the switching period is much larger and smaller than the timescale of the individual modes. Predicting the onset of an antiresonance requires the study of the nonlinear interplay between the intrinsic dynamics of each mode at intermediate switching frequencies.

Antiresonance at intermediate switching. Surprisingly, there is a wide range of intermediate switching periods that induce the stability of the switched system so that the origin becomes a stable focus (see Fig. 2). The stabilization mechanism relies on switching slow enough to be attracted toward the origin during the rotation, but fast enough to avoid reaching the unstable principal directions. For $\omega=2$ and $\delta=0.5$, simulations suggest that the range of $T$ that guarantees convergence toward the origin is $T \in(1.18,4.26)$. The emergence of this antiresonant response is completely open-loop, whereby switching takes place without knowledge of the present state of the system. Below, we prove the existence of this antiresonance window and characterize its size as a function of the system parameters.

Mathematical analysis of stability. System (1) is conveniently written in polar coordinates as $z=r \exp (i \theta)$,

$$
\begin{aligned}
\dot{r} & =r+\omega r(2 s-1) \sin 2 \theta, \\
\dot{\theta} & =\omega-\omega(2 s-1) \cos 2 \theta .
\end{aligned}
$$

This representation indicates that the nonlinearity is limited to the angular coordinate and that the system has a triangular structure, with the angular coordinate evolving independent of the radial one. This structure is amenable to a closed-form solution, which we later use to gain insight into stochastic switching.

To study the stability of the periodically-switched system, we perform the change of variable $\rho=1 / r$, so that (3a) becomes

$$
\dot{\rho}=-\rho-\omega \rho(2 s-1) \sin 2 \theta .
$$


This change of variables maps the origin to infinity and vice versa, thereby turning antiresonance of nonlinear system (1) into the resonance of an analytically-tractable linear system. Expanding on these studies, we constructively determine the range of switching frequencies that lead to antiresonance of (1) for any selection of $\delta$ and fully characterize the basin of attraction.

For $\zeta=[\rho \cos \theta, \rho \sin \theta]^{\mathrm{T}}$, we obtain the following linear switched system:

$$
\dot{\zeta}(t)=A(t) \zeta(t), \text { with } A(t)=\left[\begin{array}{cc}
-1 & -2 \omega s(t) \\
2 \omega(1-s(t)) & -1
\end{array}\right] .
$$

System (5) periodically switches between two linear modes determined by two Hurwitz matrices, $A_{0}$ when the switch is off $(s=0)$ and $A_{1}$ when the switch is on $(s=1)$, such that

$$
A_{0}=\left[\begin{array}{cc}
-1 & 0 \\
2 \omega & -1
\end{array}\right] \text { and } A_{1}=\left[\begin{array}{cc}
-1 & -2 \omega \\
0 & -1
\end{array}\right]
$$

These matrices have repeated eigenvalues $\lambda=-1$, yielding a stable degenerate node in both modes.

Similar dynamics have been considered in Refs. [23,24], in the context of stochastic switching for continuous and discrete systems, respectively. The main differences with respect to the present analysis are: (i) our analysis is for a general value of $\delta$, while the setups in Refs. [23,24] assume equal probability for the on and off states, (ii) we constructively demonstrate a compact window of stability for the switching rate and provide a closed form result for it, while Ref. [24] identifies integer values that are pertinent to discrete dynamics and Ref. [23] only points at the existence of a rate that guarantees instability, and (iii) we address the dependence on initial conditions toward the characterization of the basin of attraction of the original switched system, which is not part of the analysis in Refs. [23,24].

The stability of a linear periodically-switched system (5) can be examined using classical Floquet theory [39]. Given the transition matrix $\Phi(t, \tau)$, we compute the $T$-periodic function $P(t)$ and the constant (possibly complex) matrix $R$

$$
\begin{aligned}
R & =\frac{1}{T} \log [\Phi(T, 0)], \\
P(t) & =\Phi(t, 0) \exp (-R t) .
\end{aligned}
$$

Through $P(t)$, we define a Lyapunov transformation that converts the original switched system into an ancillary, timeinvariant one with state matrix equal to $R$ [40]. The entire stability analysis of nonlinear system (1) reduces to the study of the eigenvalues of $R$, the so-called characteristic exponents.

The linear switched system is (uniformly and exponentially) asymptotically stable if and only if all the characteristic exponents have negative real parts. The computation of the characteristic exponents requires the calculation of the two-dimensional monodromy matrix $\Phi(T, 0)$, given by $\Phi(T, 0)=\exp \left(\delta T A_{1}\right) \exp \left[(1-\delta) T A_{0}\right]$. By taking the logarithm of the matrix and computing its eigenvalues, we analytically determine the two characteristic exponents, as functions of $\delta, \omega$, and $T$ (see Fig. 3). Specifically,
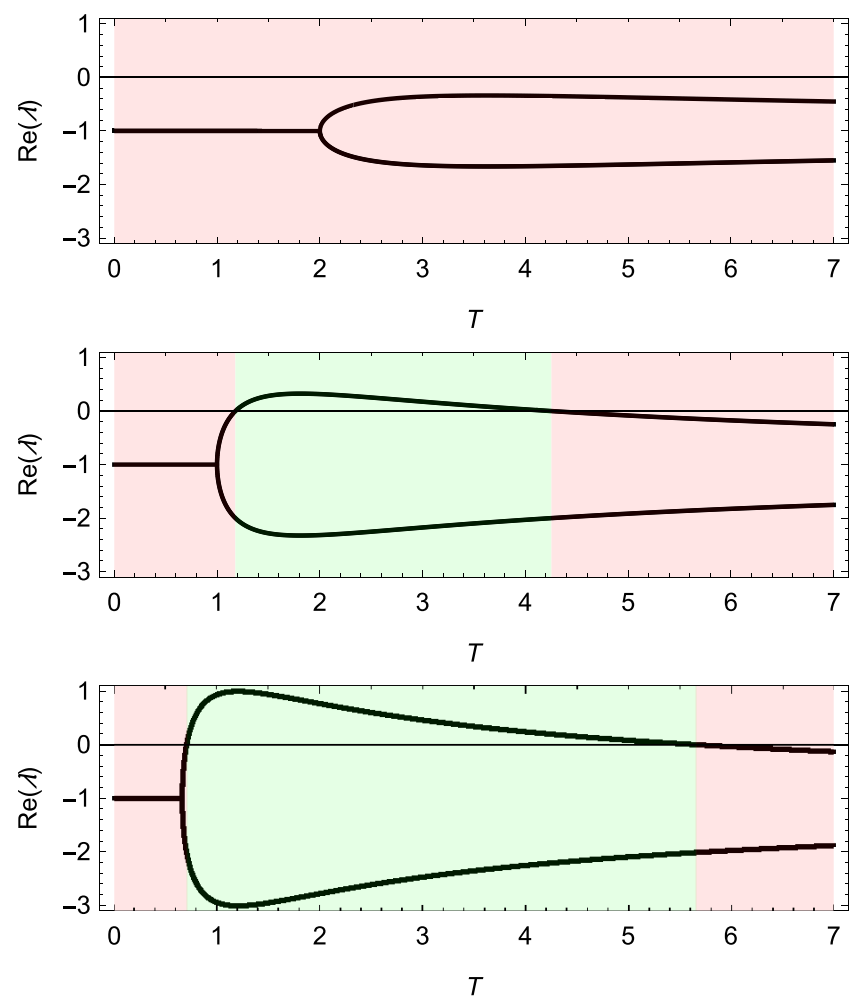

FIG. 3. Real part of the characteristic exponents of the linear periodically-switching (5) as functions of $T$ for $\delta=0.5$ and different values of $\omega: \omega=1$ (top), $\omega=2$ (middle), and $\omega=3$ (bottom). Red regions identify values for which the real parts of both the exponents are negative and green regions values for which one is positive.

we determine

$$
\begin{aligned}
\lambda= & -1-\frac{\log 2}{T}+\frac{1}{T} \log [2-4 \omega T(\delta(1-\delta) \omega T \\
& \left.\left. \pm \sqrt{\delta(1-\delta)\left(-1+\delta(1-\delta) \omega^{2} T^{2}\right)}\right)\right] .
\end{aligned}
$$

In agreement with simulations in Fig. 2, small and large values of the switching periods result into an unstable dynamics, that is, negative characteristic exponents of the ancillary, linear system [41]. For sufficiently large values of $\omega$, one of the characteristic exponents becomes positive, thereby triggering the instability of the ancillary system. Such an instability, in turn, manifests into the stable dynamics of the original nonlinear system.

There is a wide range of values of $T$ for which the original system is asymptotically stable (uniformly and exponentially) with a basin of attraction that comprises the whole phase space, except of a principal instability direction. Such a direction corresponds to the eigenvector of $R$ that is associated with the eigenvalue with negative real part. In general, for $T>1 /(\omega \sqrt{\delta(1-\delta)}), R$ has two real eigenvectors that coincide at a phase $-\arctan \sqrt{(1-\delta) / \delta}$ for $T=1 /(\omega \sqrt{\delta(1-\delta)})$ and then separate one from each other as $T$ increases. In the limit of $T \rightarrow \infty$, they approach 0 and $-\pi / 2$, with the latter corresponding to the eigenvalue with the smaller real part, that is, the principal unstable direction of the original system [42]. Within the window of stability of the original system, the principal direction will thus vary in an interval contained 
between $-\arctan \sqrt{\delta /(1-\delta)}$ and $-\pi / 2$; for example, for $\omega=1$ and $\delta=0.5$, it varies between -1.06 and -1.45 .

Extension to stochastic switching. While the proposed analytical treatment strictly applies to periodic switching, insight into the antiresonance of system (1) to more complex switching processes could be garnered by examining the polar representation in (1). Equation (3b) admits the following solution:

$$
\begin{aligned}
& s=0: \theta(t)=\arctan \left[2 \omega\left(t-t_{i}\right)+\tan \theta\left(t_{i}\right)\right], \\
& s=1: \theta(t)=-\operatorname{arccot}\left[2 \omega\left(t-t_{i}\right)+\cot \theta\left(t_{i}\right)\right],
\end{aligned}
$$

where $t_{i}$ is the instant of switching.

With knowledge of the time evolution of the angular coordinate in (9), we can solve (3a) for the radial coordinate to obtain

$$
\begin{aligned}
& s=0: \frac{r(t)}{r\left(t_{i}\right)}=\exp \left(t-t_{i}\right) \sqrt{\frac{1+\tan ^{2} \theta\left(t_{i}\right)}{1+\left(2 \omega\left(t-t_{i}\right)+\tan \theta\left(t_{i}\right)\right)^{2}}}, \\
& s=1: \frac{r(t)}{r\left(t_{i}\right)}=\exp \left(t-t_{i}\right) \sqrt{\frac{1+\cot ^{2} \theta\left(t_{i}\right)}{1+\left(2 \omega\left(t-t_{i}\right)-\cot \theta\left(t_{i}\right)\right)^{2}}},
\end{aligned}
$$

where we made evident the linearity by factoring the initial condition on the left-hand-sides of the equations.

Equations (9) and (10) are valid for any choice of the system parameters and switching process. As an example of stochastic switching, we consider the case where we retain the same switching instants as the periodic process, but the value of $s(t)$ is drawn with equal probability between 0 and 1 (this approach could also be adapted to study Markovian switching processes [43]). We simulate (9) and (10) for 1000 switches and 1000 realizations of the stochastic switching sequences. The initial angle $\theta(0)$ is drawn uniformly at random from 0 to $2 \pi$ and convergence is ascertained by comparing the initial with the final radius.

Predictably, for small values of $\omega$, we do not obtain the spiraling trajectories that are required for the stabilization of the origin, thereby hindering antiresonance through stochastic switching (see Fig. 4). For larger values of $\omega$, we observe the onset of antiresonance through stochastic switching, but the range of antiresonance periods considerably narrows with respect to the periodic case [44]. This phenomenon is due to the nonlinearity of the system, which reduces the set of favorable switching sequences as the period increases.

Conclusions. Stabilization of unstable systems via periodic or stochastic perturbations is a critical problem in nonlinear dynamics, with applications in physics, biology, and engineering. Just as an oscillating suspension can stabilize the inverted configuration of the classical Kapitza's pendulum [45], vibrational stabilization is used in hovering insects and flapping wing micro-air vehicles [46]. Common to these examples is the existence of some stable dynamics that could facilitate the stabilization of the system.

Surprisingly, the same phenomenon can be observed without any stable dynamics to fall back on, like in the case of synchronization in temporal networks of Rössler or Duffing oscillators [47] and prey-predator metapopulations [48]. Simulations revealed the existence of windows of opportunity for the time scale of the network evolution, where the syn-
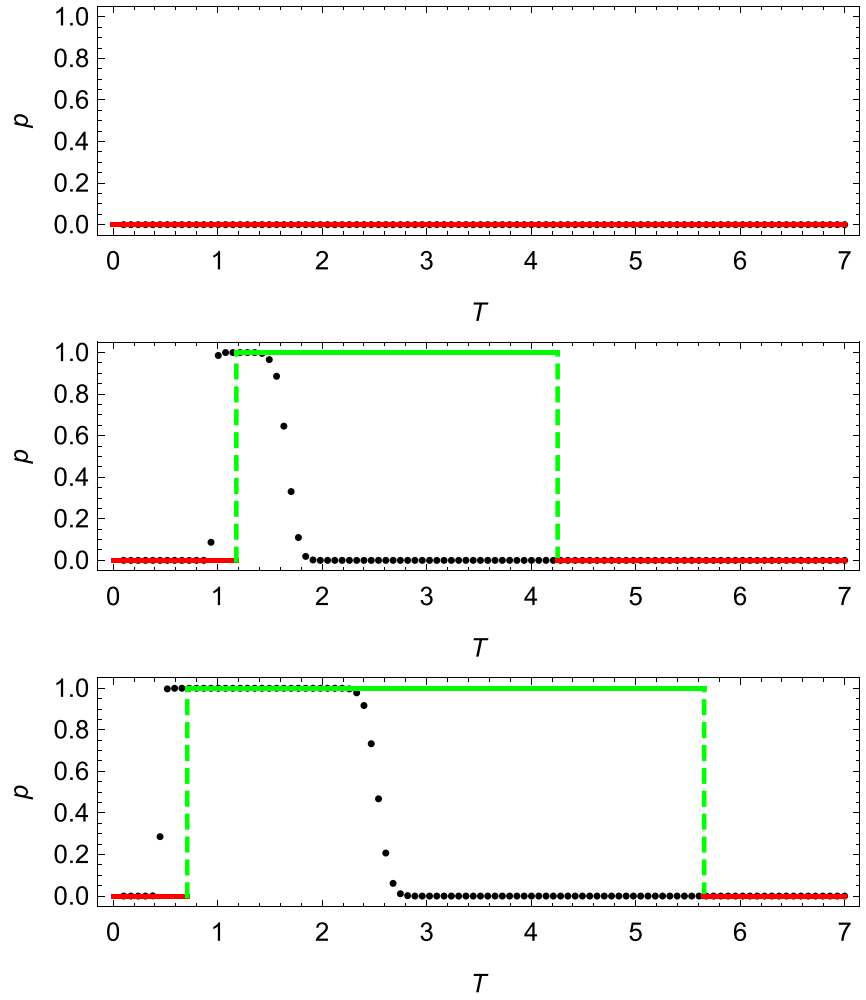

FIG. 4. Probability of converging to the origin for stochastic versus periodic switching in system (1) as functions of $T$ for $\delta=0.5$ and different values of $\omega: \omega=1$ (top), $\omega=2$ (middle), and $\omega=3$ (bottom). Black dots correspond to stochastic switching, and solid lines to periodic switching. Color coding follows Fig. 3: red and green lines identify values of $T$ for which periodic switching leads to instability and stability, respectively.

chronous state can be stabilized. Whether this phenomenon was due to the high-dimensional, nonautonomous nature of the variational equations describing the error dynamics or to a more profound structural property of the chaotic coupled dynamics has remained elusive.

Here, we offered compelling evidence in favor of a structural property that should underlie the stabilization phenomenon. We provided the first analytically-tractable example of antiresonance in a two-dimensional switched system with only two unstable modes, described by variation of a StuartLandau model. Remarkably, the average dynamics of the switched system is unstable, thereby prohibiting stabilization through fast-switching, and the unstable dynamics of each mode ensures instability at low switching frequencies. Yet, the system acquires stability in a range of intermediate switching frequencies.

Our example indicates that stabilization is possible because of a hybrid type of unstable dynamics, characterized by a fixed point that has features of an unstable node and a stable focus. This structural property is common to saddle-focus dynamics of Rössler, Duffing, and prey-predator models, which display an equivalent behavior in higher dimensions. We conjecture that this dynamics is responsible for a latent stability that can be effectively explored while switching in the antiresonance frequency range, thereby supporting the emergence of windows of opportunity in synchronization problems. In loose 
terms, the stabilization mechanism can be explained using the analogy of juggling a hot potato between two hands: The system must spend some time in one mode (one hand) to take advantage of its latent stability property (the time it takes to acquire a firm control of the potato) and then it must leave this mode before its inherent instability manifests (before the hand gets burned).

The simplicity and richness of our system make the case for it to become a nonlinear dynamics textbook example of "impossible" stable dynamics emerging from antiresonant switching between unstable modes. We hope that our system can find a home close to some illustrious, two-dimensional cases of counterintuitive nonlinear dynamics, like Vinograd's example of a system that is unstable but convergent to the origin [49].

Acknowledgments. The authors thank B. Behring for help in verifying analytical results and A. Boldini for useful discussions. This work was supported by the National Science Foundation (USA) under Grants No. CMMI-1561134 and No. CMMI-1932187 (to M.P.), No. DMS-1909924 (to R.J. and I.B.) and No. CMMI-2009329 (to I.B.), and by the Ministry of Science and Higher Education of the Russian Federation under Project No. 0729-2020-0036 (to I.B.).
[1] D. Liberzon, Switching in Systems and Control (Springer Science \& Business Media, New York, 2012).

[2] S. Camazine, J.-L. Deneubourg, N. R. Franks, J. Sneyd, E. Bonabeau, and G. Theraulaz, Self-Organization in Biological Systems (Princeton University Press, Princeton, NJ, 2003), Vol. 7.

[3] P. Holme and J. Saramäki, Temporal networks, Phys. Rep. 519, 97 (2012).

[4] D. Needleman and Z. Dogic, Active matter at the interface between materials science and cell biology, Nat. Rev. Mater. 2, 17048 (2017).

[5] O. Feinerman, I. Pinkoviezky, A. Gelblum, E. Fonio, and N. S. Gov, The physics of cooperative transport in groups of ants, Nat. Phys. 14, 683 (2018).

[6] P. S. Churchland and T. J. Sejnowski, The Computational Brain (MIT Press, Cambridge, MA, 2016).

[7] C. K. Tse and M. Di Bernardo, Complex behavior in switching power converters, Proc. IEEE 90, 768 (2002).

[8] M. J. O'Mahony, D. Simeonidou, D. K. Hunter, and A. Tzanakaki, The application of optical packet switching in future communication networks, IEEE Commun. Mag. 39, 128 (2001).

[9] Z. Sun and S. S. Ge, Stability Theory of Switched Dynamical Systems (Springer Science \& Business Media, New York, 2011).

[10] H. Lin and P. J. Antsaklis, Stability and stabilizability of switched linear systems: a survey of recent results, IEEE Trans. Autom. Control 54, 308 (2009).

[11] N. Agarwal, A simple loop dwell time approach for stability of switched systems, SIAM J. Appl. Dyn. Syst. 17, 1377 (2018).

[12] G. Zhai, Bo Hu, K. Yasuda, and A. N. Michel, Stability analysis of switched systems with stable and unstable subsystems: an average dwell time approach, Int. J. Syst. Sci. 32, 1055 (2001).

[13] E. Mostacciuolo, S. Trenn, and F. Vasca, Averaging for switched DAEs: Convergence, partial averaging and stability, Automatica 82, 145 (2017).

[14] I. Belykh, V. Belykh, R. Jeter, and M. Hasler, Multistable randomly switching oscillators: The odds of meeting a ghost, Eur. Phys. J.: Spec. Top. 222, 2497 (2013).

[15] Y. Bakhtin, T. Hurth, S. D. Lawley, and J. C. Mattingly, Smooth invariant densities for random switching on the torus, Nonlinearity 31, 1331 (2018).

[16] M. Frasca, A. Buscarino, A. Rizzo, L. Fortuna, and S. Boccaletti, Synchronization of Moving Chaotic Agents, Phys. Rev. Lett. 100, 044102 (2008).
[17] T. E. Gorochowski, M. di Bernardo, and C. S. Grierson, Evolving enhanced topologies for the synchronization of dynamical complex networks, Phys. Rev. E 81, 056212 (2010).

[18] V. Kohar, P. Ji, A. Choudhary, S. Sinha, and J. Kurths, Synchronization in time-varying networks, Phys. Rev. E 90, 022812 (2014).

[19] D. J. Stilwell, E. M. Bollt, and D. G. Roberson, Sufficient conditions for fast switching synchronization in timevarying network topologies, SIAM J. Appl. Dyn. Syst. 5, 140 (2006).

[20] A. Mondal, S. Sinha, and J. Kurths, Rapidly switched random links enhance spatiotemporal regularity, Phys. Rev. E 78 , 066209 (2008).

[21] P. So, B. C. Cotton, and E. Barreto, Synchronization in interacting populations of heterogeneous oscillators with time-varying coupling, Chaos 18, 037114 (2008).

[22] P. C. Bressloff and S. D. Lawley, Hybrid colored noise process with space-dependent switching rates, Phys. Rev. E 96, 012129 (2017).

[23] S. D. Lawley, J. C. Mattingly, and M. C. Reed, Sensitivity to switching rates in stochastically switched ODEs, Commun. Math. Sci. 12, 1343 (2014).

[24] M. Porfiri, R. Jeter, and I. Belykh, Windows of opportunity for the stability of jump linear systems: almost sure versus moment convergence, Automatica 100, 323 (2019).

[25] A. A. Andronov, A. A. Vitt, and S. E. Khaikin, Theory of Oscillators: Adiwes International Series in Physics (Elsevier, Amsterdam, 2013), Vol. 4.

[26] W. Greiner, Quantum Mechanics: An Introduction (Springer Science \& Business Media, New York, 2011).

[27] L. Canetti, M. Drewes, and M. Shaposhnikov, Matter and antimatter in the universe, New J. Phys. 14, 095012 (2012).

[28] G. Ma, M. Yang, S. Xiao, Z. Yang, and P. Sheng, Acoustic metasurface with hybrid resonances, Nat. Mater. 13, 873 (2014).

[29] D. Plankensteiner, C. Sommer, H. Ritsch, and C. Genes, Cavity Antiresonance Spectroscopy of Dipole Coupled Subradiant Arrays, Phys. Rev. Lett. 119, 093601 (2017).

[30] J. D. Touboul, C. Piette, L. Venance, and G. B. Ermentrout, Noise-Induced Synchronization and Antiresonance in Interacting Excitable Systems: Applications to Deep Brain Stimulation in Parkinson's Disease, Phys. Rev. X 10, 011073 (2020).

[31] C. Sames, H. Chibani, C. Hamsen, P. A. Altin, T. Wilk, and G. Rempe, Antiresonance Phase Shift in Strongly Coupled Cavity QED, Phys. Rev. Lett. 112, 043601 (2014). 
[32] In Cartesian coordinates, the system reads as $s=0: \dot{x}=x-$ $4 \omega x^{2} y /\left(x^{2}+y^{2}\right), \dot{y}=y+2 \omega\left(x^{3}-x y^{2}\right) /\left(x^{2}+y^{2}\right)$ and $s=1:$ $\dot{x}=x-2 \omega\left(y^{3}-x^{2} y\right) /\left(x^{2}+y^{2}\right), \dot{y}=y+4 \omega x y^{2} /\left(x^{2}+y^{2}\right)$.

[33] V. García-Morales and K. Krischer, The complex GinzburgLandau equation: An introduction, Contemp. Phys. 53, 79 (2012).

[34] A classical Stuart-Landau oscillator has $f\left(z, z^{*}\right) \propto z^{*} z^{2}$, which is the simplest nonlinearity to guarantee rotational invariance.

[35] N. Fujiwara, T. Kobayashi, and H. Fujisaka, Dynamic phase transition in a rotating external field, Phys. Rev. E 75, 026202 (2007).

[36] A. Zakharova, M. Kapeller, and E. Schöll, Chimera Death: Symmetry Breaking in Dynamical Networks, Phys. Rev. Lett. 112, 154101 (2014).

[37] D. V. R. Reddy, A. Sen, and G. L. Johnston, Dynamics of a limit cycle oscillator under time delayed linear and nonlinear feedbacks, Physica D 144, 335 (2000).

[38] O. V. Popovych, C. Hauptmann, and P. A. Tass, Effective Desynchronization by Nonlinear Delayed Feedback, Phys. Rev. Lett. 94, 164102 (2005).

[39] W. J. Rugh, Linear System Theory (Prentice-Hall, Inc., Upper Saddle River, NJ, 1996).

[40] By construction, matrix $P(t)$ and its inverse are bounded and piecewise continuously differentiable, thereby preserving stability properties in a Lyapunov transformation. Hence, we specifically set $\hat{\zeta}(t)=P^{-1}(t) \zeta(t)$, which leads to $\hat{\zeta}(t)=R \hat{\zeta}(t)$.
[41] For $T \ll 1$, the system behaves like the average system (1 $\delta) A_{0}+\delta A_{1}$, which has eigenvalues $-1 \pm 2 i \omega \sqrt{(1-\delta) \delta}$; for $T \gg 1$, the system behaves like the individual modes that have eigenvalues all equal to -1 .

[42] In the slow-switching limit, $R \rightarrow-I$, where $I$ is the twodimensional identity matrix.

[43] M. Porfiri and I. Belykh, Memory matters in synchronization of stochastically coupled maps, SIAM J. Appl. Dyn. Syst. 16, 1372 (2017).

[44] Should one consider the case in which switching occurs every $T$ time units with equal probability, the range of antiresonance would be even smaller.

[45] P. L. Kapitza, A pendulum with oscillating suspension, Usp. Fiz. Nauk 44, 7 (1951).

[46] H. Taha, M. Kiani, and J. Navarro, Experimental demonstration of the vibrational stabilization phenomenon in bioinspired flying robots, IEEE Robot. Autom. Lett. 3, 643 (2017).

[47] R. Jeter and I. Belykh, Synchronization in on-off stochastic networks: windows of opportunity, IEEE Trans. Circuits Syst. I: Reg. Papers 62, 1260 (2015).

[48] R. Jeter and I. Belykh, Synchrony in metapopulations with sporadic dispersal, Int. J. Bifurcation Chaos 25, 1540002 (2015).

[49] W. Hahn, Stability of Motion (Springer, New York, 1967), Vol. 138. 